\title{
Avaliação Fisioterapêutica Aquática para Crianças: Uma Revisão Integrativa
}

\author{
Mariane Borato, ${ }^{1}$ Stephany Ferreira de Souza, ${ }^{2}$ Jessica Biernaski, ${ }^{2}$ \\ Deborah Fernandes Lucas, ${ }^{2}$ Beatriz Komar de Carvalho, ${ }^{2}$ Tainá Ribas Melo, ${ }^{2}$ \\ Luize Bueno de Araujo, ${ }^{2}$ Vera Lúcia Israel ${ }^{2}$
}

\begin{abstract}
RESUMO
O processo de desenvolvimento humano é complexo, dinâmico e necessita de atenção especial durante a infância. Em decorrência disso, são necessários métodos avaliativos fisioterapêuticos a fim de monitorar, mensurar e comparar a evolução desses comportamentos e habilidades motoras. A Fisioterapia Aquática em crianças cria diversas possibilidades de vivências e aprendizados em meio líquido, permitindo uma abordagem lúdica. O objetivo desta pesquisa é rastrear na literatura estudos de programas de intervenções aquáticas, buscando identificar métodos de avaliação que foram utilizados e aplicados em crianças tanto em ambiente aquático quanto em solo, por meio de uma revisão integrativa. Trata-se de um artigo de revisão integrativa, em que os artigos foram selecionados pelas permutas de palavras-chave que ocorreram na base de dados Biblioteca Virtual em Saúde (BVS). Foram encontrados 34 artigos e, após exclusão, destinaram-se seis artigos para a leitura na íntegra, pois atendiam aos critérios. Como produto final, encontrou-se apenas um artigo com método avaliativo aquático, evidenciando uma carência de métodos avaliativos neste ambiente, principalmente para as crianças, o que identifica a necessidade de maiores estudos sobre novas formas de avaliação aquática.
\end{abstract}

Palavras-chave: Fisioterapia. Hidroterapia. Crianças. Avaliação.

\section{AQUATIC PHYSIOTHERAPY ASSESSMENT FOR CHILDREN: AN INTEGRATIVE REVIEW}

\section{ABSTRACT}

The human development process is complex, dynamic, and needs special attention during childhood. As a result, physiotherapeutic methods of evaluation are necessary to monitor, measure, and compare the evolution of comportments and motor skills. Diverse possibilities of experiences and learning in watery are created by Aquatic Physiotherapy with children, allowing a playful approach. The objective of this research is to track studies of aquatic intervention programs in the literature, seeking to identify assessment methods that have been used and applied to children, both in the aquatic environment and on the ground, through integrative review. This paper's objective is to search studies of aquatic intervention programs in literature and identify evaluation methods in water or soil. The paper is an integrative review article in which the articles were selected by keywords exchange that occurred in the Virtual Health Library (VHL) database. The search found 34 papers, of which six met the criteria, and those were the articles selected for reading in full. After reading, one document had aquatic evaluation as a subject, which evidences a lack of evaluation methods in this environment, mainly with children, pointing out the need for studies about new forms of aquatic assessment.

Keywords: Physical therapy. Hydrotherapy. Child. Assessment.

RECEBIDO EM: 30/9/2020

MODIFICAÇÕES SOLICITADAS EM: 13/11/2020

ACEITO EM: 5/1/2021

\footnotetext{
${ }^{1}$ Autora correspondente. Universidade Federal do Paraná - UFPR. Rua XV de Novembro, 1299 - Centro. Curitiba/PR, Brasil. CEP 80060-000. http://lattes.cnpq.br/5394181579920225. https://orcid.org/0000-0001-6091-2034. marianeborato@gmail.com

2 Universidade Federal do Paraná - UFPR. Curitiba/PR, Brasil.
} 


\section{INTRODUÇÃO}

Uma das características do ser humano é estar em um processo constante de desenvolvimento, a contar do seu nascimento até o fim da vida (GUERREIRO et al., 2016). O desenvolvimento neuropsicomotor (DNPM) é complexo, dinâmico e possui influências de diversos fatores, entre eles o contexto em que a criança está inserida, tanto ambiente físico quanto social (HWANG et al., 2014). O comportamento motor, portanto, deve ser considerado em todas as suas reações reflexas, voluntárias e aprendidas, compreendendo-o como um modo que atinge cinco grandes áreas do desenvolvimento humano: motor grosseiro, motor fino/ adaptativo, pessoal/social, linguagem e cognitivo (NAZARIO; ARINS; KURZ, 2016).

Para conhecer o DNPM e os contextos envolvidos na estimulação da criança, são necessárias pesquisas sistematizadas para organizar os processos de profissionais de diferentes áreas da educação e da saúde. Dentro destes processos, um dos grandes desafios é a avaliação do DNPM na busca de possibilidades de explicar como este ocorre e suas transformações decorrentes da própria maturação e sua abrangência contextual global (BARBA, 2007). No caso da fisioterapia, a avaliação fisioterapêutica consegue traçar objetivos terapêuticos e funcionais, de promoção, prevenção e recuperação cinético-funcional, permitindo uma intervenção individualizada que pode potencializar o desenvolvimento ou a reabilitação da criança (GAVIM et al., 2013).

Dentro do processo fisioterapêutico é possível escolher vários recursos para trazer benefícios ao DNPM e, no caso dessa revisão integrativa, a abordagem escolhida foi a Fisioterapia Aquática (FA). A FA apropria-se da piscina aquecida e das propriedades físicas e térmicas deste ambiente para garantir vivências e aprimorar a percepção sensorial e ação motora da criança (IUCKSCH et al., 2020). Além de ser um espaço privilegiado para estimular a criança, a terapia, nesse ambiente, envolve comportamentos das áreas cognitivas de personalidade, linguagem, áreas motoras, como equilíbrio corporal e estabilidade postural, entre outros. Pode-se induzir, portanto, adaptações funcionais e sociais em bebês por meio do contato humano, permitindo que o desenvolvimento infantil ocorra de forma gradual e natural (SILVA et al., 2009).

A prática clínica deve estar fundamentada sob as evidências científicas. Dessa forma, deve-se buscar quantificar resultados obtidos em todos os locais de atuação fisioterapêutica. Com relação à avaliação em ambiente aquático, há a possibilidade de se identificar qual o nível de controle corporal existente, monitorar a evolução dos pacientes e verificar a assertividade da intervenção proposta. Como exemplo, pode-se citar a Aquatic Functional Assessment Scale (Afas), que objetiva classificar a adaptação e a independência de indivíduos adultos na água (ISRAEL; PARDO, 2014).

De acordo com Araujo et al. (2019), ainda há poucos estudos que evidenciam/avaliam o comportamento de indivíduos no ambiente aquático, justificando esta investigação, uma vez que pesquisas demonstram que intervenções aquáticas com crianças utilizam as mesmas avaliações de terapias em solo para examinar e monitorar as evoluções, não sendo algo específico para o ambiente em que a terapia está inserida (NOVAKOSKI et al., 2018).

Editora Unijuí - Revista Contexto \& Saúde - ISSN 2176-7114 - v. 21, n. 42, jan./jun. 2021 
Desta forma, o objetivo desta pesquisa é rastrear na literatura estudos de programas de intervenções aquáticas, buscando identificar métodos de avaliação que foram utilizados e aplicados em crianças, tanto em ambiente aquático quanto em solo, por meio de uma revisão integrativa.

\section{METODOLOGIA}

Este estudo caracteriza-se como uma revisão integrativa (NOVAKOSKI et al., 2018; SOUZA; SILVA; CARVALHO, 2010), que se trata de uma síntese de pesquisas que abordam o mesmo tema, com o intuito de encontrar publicações e autores que descrevem e/ou citam o uso de métodos de avaliação aquática para crianças. A base de dados escolhida para buscar os artigos foi a Biblioteca Virtual em Saúde (BVS, 1998), incluindo Lilacs, Medline e Pubmed.

Os critérios de inclusão do estudo foram artigos disponíveis na plataforma BVS no período de janeiro de 2015/fevereiro de 2020, respeitando o limite de cinco anos desde a data de publicação. Além disso, o idioma (português-inglês), o tipo de estudo (ensaio clínico) e a disponibilidade do texto completo de forma gratuita, foram também critérios de inclusão.

As buscas foram realizadas pelas pesquisadoras no período de janeiro-fevereiro de 2020. Entre os descritores foi usado o AND (para unir termos), OR (para separar palavras com o mesmo significado) e para palavras compostas foram usadas as aspas ("). As palavras-chave escolhidas para concretizar as buscas foram: aquatic, child $O R$ baby $O R$ babies $O R$ infant, evaluation OR assessment, exercise, hydrotherapy, motor skill, physiotherapy OR physical therapy, swimming.

A primeira combinação foi realizada com seguintes palavras-chave: "physiotherapy OR physical therapy" AND "child OR baby OR babies OR infant" AND "evaluation OR assessment" AND hydrotherapy, e, após essa etapa, foram realizadas combinações de três palavras, sendo fixadas "child OR baby OR babies $O R$ infant" AND "hydrotherapy", alternando o terceiro componente da combinação. O método é exemplificado na Tabela 1.

Tabela 1 - Descritores utilizados na busca

\begin{tabular}{llllcc}
\hline \multicolumn{1}{c}{ PALAVRAS-CHAVE FIXAS } & & $\begin{array}{c}\text { PALAVRAS } \\
\text { ALTERNADAS }\end{array}$ & $\begin{array}{c}\text { QUANTIDADE } \\
\text { DE ARTIGOS } \\
\text { ENCONTRADOS }\end{array}$ \\
\hline $\begin{array}{c}\text { Child OR Baby } \\
\text { OR Babies OR } \\
\text { Infant }\end{array}$ & AND & Hydrotherapy & AND & $\begin{array}{c}\text { Physiotherapy OR } \\
\text { physical therapy }\end{array}$ & 7 \\
$\begin{array}{c}\text { Child OR Baby } \\
\text { OR Babies OR } \\
\text { Infant }\end{array}$ & AND & Hydrotherapy & AND & $\begin{array}{c}\text { Evaluation OR } \\
\text { Assessment }\end{array}$ & 3 \\
$\begin{array}{c}\text { Child OR Baby } \\
\text { OR Babies OR } \\
\text { Infant }\end{array}$ & AND & Hydrotherapy & AND & Motor Skill & 1 \\
$\begin{array}{c}\text { Child OR Baby } \\
\text { OR Babies OR } \\
\text { Infant }\end{array}$ & AND & Hydrotherapy & AND & Exercise & \\
& & & & & 4 \\
\end{tabular}




\begin{tabular}{|c|c|c|c|c|c|}
\hline $\begin{array}{c}\text { Child OR Baby } \\
\text { OR Babies OR } \\
\text { Infant }\end{array}$ & AND & Hydrotherapy & AND & Aquatic & 4 \\
\hline $\begin{array}{c}\text { Child OR Baby } \\
\text { OR Babies OR } \\
\text { Infant }\end{array}$ & AND & Hydrotherapy & AND & Swimming & 0 \\
\hline
\end{tabular}

Fonte: As autoras.

Com isto, após as buscas foi realizada a exclusão dos artigos de acordo com a sequência: título, resumo e o texto, propriamente. A seleção dos títulos contemplou crianças em um ambiente aquático. Com relação ao resumo, a exclusão deu-se por não abordar assuntos relacionados ao objetivo da pesquisa.

\section{RESULTADOS}

Somando-se todas as buscas feitas na base de dados BVS, foram encontrados 34 artigos. Após a leitura dos títulos dos artigos, notou-se que 19 deles se repetiram nas diferentes buscas, e outros nove foram excluídos pelo título e resumo ou não preenchiam os critérios do estudo, como já citado. Com isto, destinaram-se seis artigos para a leitura na íntegra, pois atendiam aos critérios inicialmente propostos (Figura 1).

Figura 1 - Fluxograma de seleção dos artigos

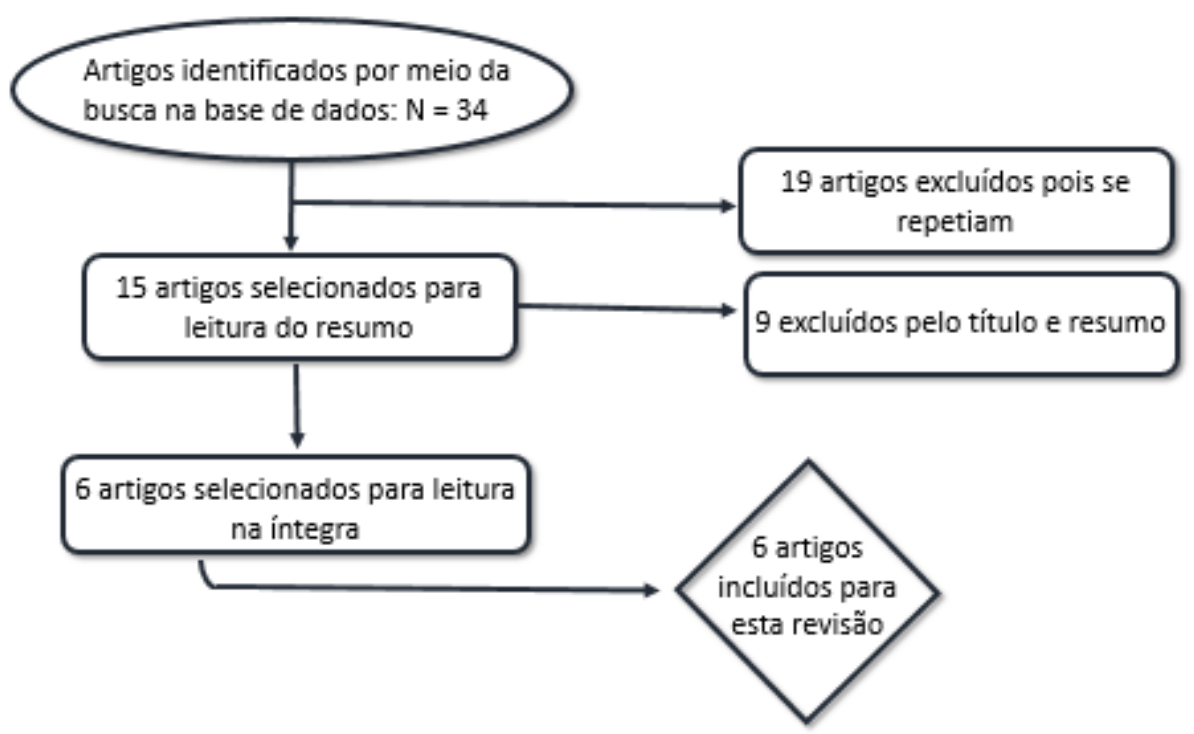

Fonte: As autoras.

Na Tabela 2 estão descritos os seis artigos finais selecionados, apresentando as categorias, nome do artigo, autor/ano de publicação, objetivo do estudo, público-alvo e desfecho do estudo. 


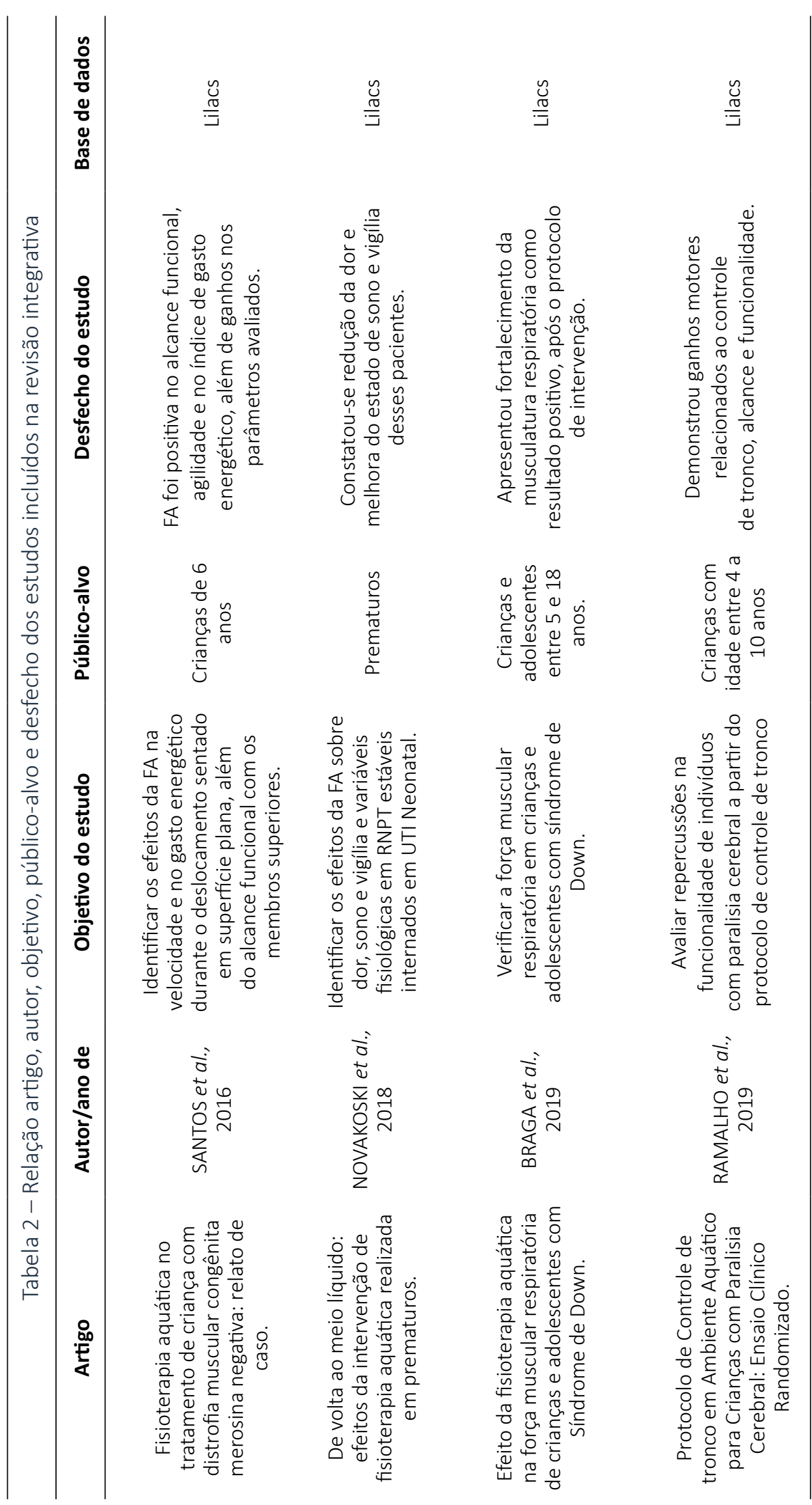

Contexto Saúde 


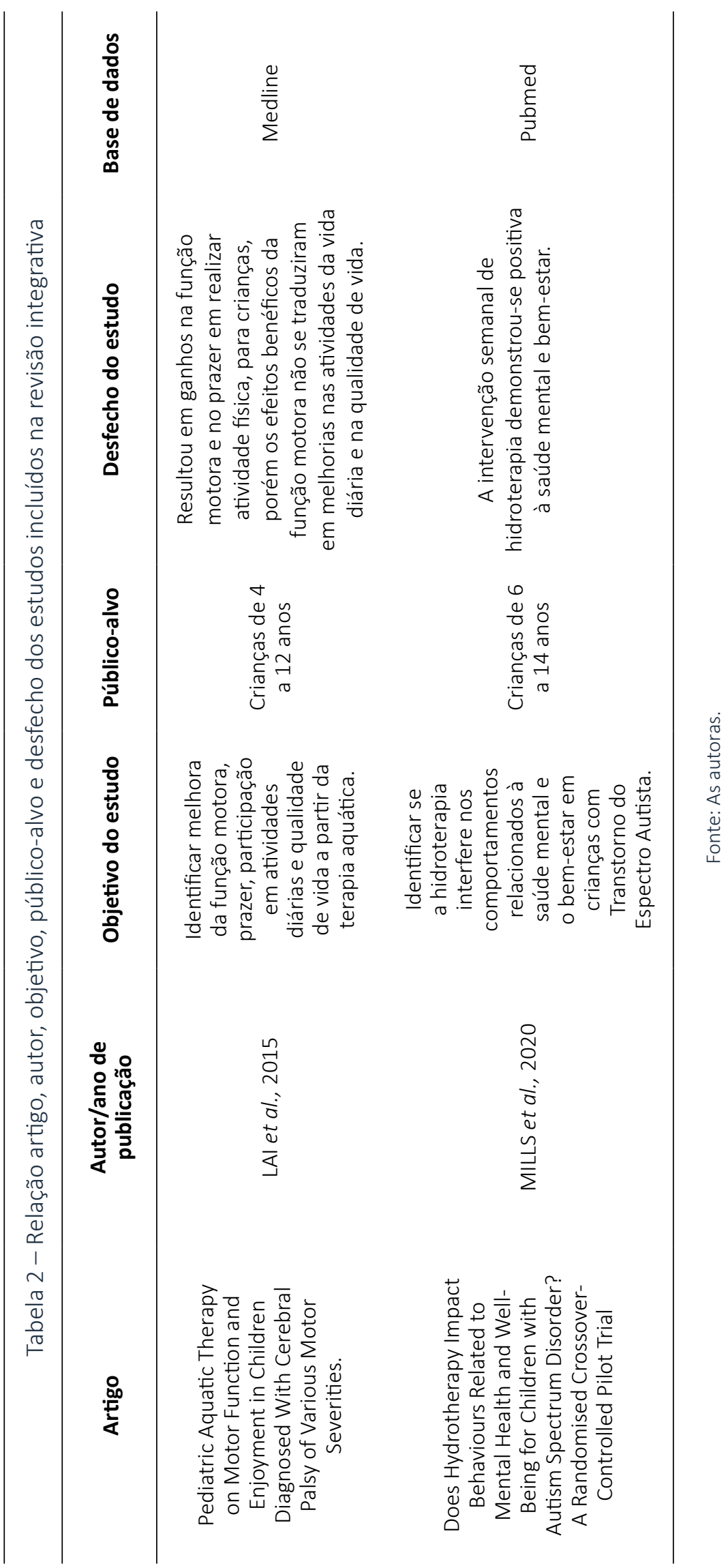

Editora Unijuí - Revista Contexto \& Saúde - ISSN 2176-7114 - v. 21, n. 42, jan./jun. 2021 
Ainda em relação aos artigos filtrados, apesar de utilizarem a Fisioterapia aquática (FA) como forma de intervenção, há diferenças entre eles; dentre estas, tem-se a escolha dos métodos avaliativos utilizados, e cada estudo selecionou métodos específicos de acordo com a condição de saúde analisada. Há, porém, algo em comum no raciocínio dos autores, uma vez que houve ênfase apenas para a avaliação em solo, não incluindo possíveis métodos de avaliação aquática. Dessa forma, apenas um artigo - Santos et al. (2016) - demonstrou/citou algum método avaliativo em meio aquático: a eletromiografia subaquática.

\section{NII
Contexto
Saúde}




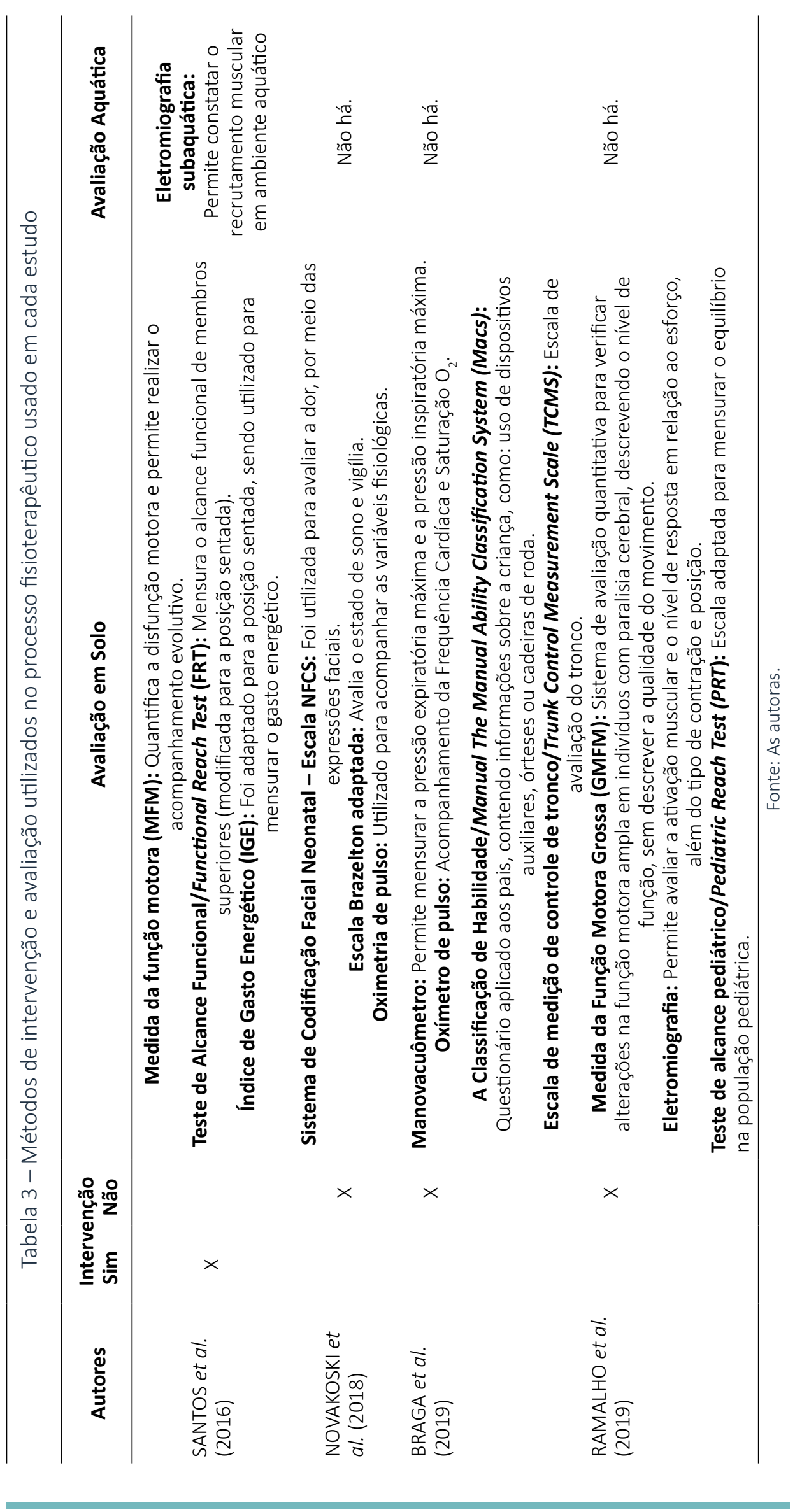

Editora Unijuí - Revista Contexto \& Saúde - ISSN 2176-7114 - v. 21, n. 42, jan./jun. 2021 


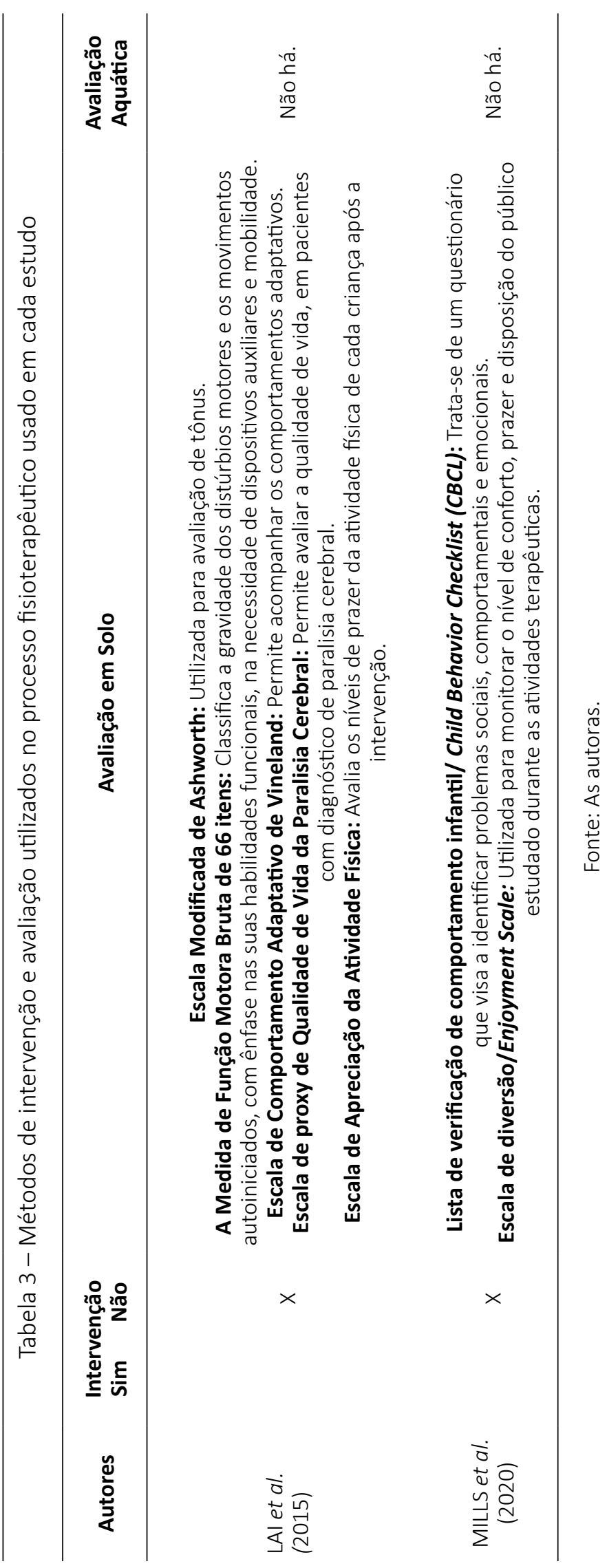

Editora Unijuí - Revista Contexto \& Saúde - ISSN 2176-7114 - v. 21, n. 42, jan./jun. 2021 


\section{DISCUSSÃO}

Com base nos estudos encontrados referentes a programas de intervenção fisioterapêutica aquática, percebe-se que há um predomínio do uso de métodos avaliativos destinados para a avaliação em solo, antes e depois da aplicação dos programas. Assim, a avaliação aquática, que traz habilidades motoras de crianças, apesar de ser imprescindível, não tem sido utilizada. Isso fundamenta-se por conta de que, nos seis artigos utilizados nesta revisão, apenas Santos et al. (2016) preocupou-se em utilizar um método de avaliação aquático, porém baseando-se na eletromiografia subaquática. Esse único método aquático avaliativo encontrado permite medir o recrutamento muscular (SANTOS et al., 2016), mas não aborda a presença de habilidades motoras aquáticas, focando especificamente nos domínios de função e estrutura, o que não abrange completamente todas os pontos relacionados à funcionalidade conforme a Classificação Internacional de Funcionamento, Incapacidade e Saúde (CIF) (MÉLO et al., 2019).

A avaliação aquática permite apurar o conhecimento sobre o corpo imerso, de forma que seja possível entender e usar melhor as propriedades da água na construção do plano de tratamento, além de conhecer quais são os níveis de controle respiratório ou presença de algum tipo de medo relacionado à água. Dessa forma, a FA objetiva o ganho de habilidades motoras, as quais devem ser transferidas ao solo, fazendo-se necessário avaliações neste meio para acompanhar os ganhos da terapia e da funcionalidade do indivíduo (IUCKSCH et al., 2020; YAMAGUCHI, 2016).

O artigo de Israel e Pardo (2014) demonstra um método avaliativo aquático funcional, com a finalidade de avaliar comportamentos motores em meio aquático, posto que tal avaliação é dividida em cinco fases: Ambientação (A), Domínio do Meio Líquido (D), Relaxamento (R), Exercícios Terapêuticos Especializados (E) e Condicionamento Orgânico Global (Cd) (ISRAEL, 2000). O estudo cita, também, outras formas de avaliação encontradas na literatura, mas, assim como em nosso estudo, a maioria detinha-se a avaliações realizadas somente em solo, como questionários, eletromiografia, escalas para mensuração da espasticidade e aspectos fisiológicos.

O pressuposto para a não utilização da avaliação em ambiente aquático se dá pela carência de equipamentos adaptados ao meio líquido que facilitem e/ou permitam tal avaliação. Estes equipamentos apresentam, muitas vezes, um custo elevado, tornando-se inviáveis à prática clínica. Estas ferramentas, contudo, fazem a diferença na aplicação dos métodos de avaliação já existentes. Além disso, outros equipamentos adaptados são necessários para o registro e controle das atividades propostas e seus resultados, de forma qualitativa e quantitativa. Para isso, é necessário a filmagem dos comportamentos motores observados durante a sessão (ISRAEL; PARDO, 2014).

Essa carência de métodos avaliativos, portanto, pode prejudicar a busca de maiores e melhores evidências sobre a intervenção fisioterapêutica em ambiente aquático, posto que a avaliação reúne dados, características, habilidades funcionais e informações capazes de conduzir a prescrição de exercícios de forma adequada a cada paciente, respeitando suas individualidades e ajustando a

Editora Unijuí - Revista Contexto \& Saúde - ISSN 2176-7114 - v. 21, n. 42, jan./jun. 2021 
intervenção para as condições específicas de cada um. Além disso, a avaliação permite o planejamento da intervenção, definindo os objetivos terapêuticos e a quantificação e qualificação das melhoras funcionais, tornando possível acompanhar a evolução do paciente (BARBOSA et al., 2006; ISRAEL; PARDO, 2014).

Apesar de essa revisão integrativa levar a seis estudos principais e apenas um que cita método avaliativo dentro da água, sua relevância em termos de levantamento permite identificar uma lacuna para a fisioterapia aquática e sugestão de estudos de validação de instrumentos de avaliação que considerem as peculiaridades desse ambiente. De acordo com Santos et al. (2016), é difícil conseguir instrumentos eficazes para mensurar com precisão cada movimento corporal na água. Também, deve ser levado em conta que as propriedades físicas e térmicas da água favorecem alguns dos movimentos corporais ou habilidades motoras (ISRAEL, 2000).

Para um bom desenvolvimento de programas de intervenção aquática, é necessário conhecer as habilidades motoras na água, principalmente em crianças. Estudo de Choi (2016) mostra que a estimulações no meio aquático, por intermédio de suas propriedades físicas e térmicas, favorecem a realização de atividades e movimentos, e podem propiciar a facilitação na realização no ambiente terrestre. Assim, os possíveis ganhos físico-funcionais no meio aquático exercem um papel significativo na manutenção das articulações e na redução da tensão muscular e da dor em várias patologias (PLECASH; LEAVITT, 2014).

Com isto, o estudo apresentou como principal limitação a indisponibilidade de artigos científicos gratuitos nas bases de dados utilizadas para o levantamento. Como lacuna observou-se a baixa quantidade de artigos na área de Fisioterapia Aquática com o intuito de identificar avaliações de habilidades motoras aquáticas em crianças.

\section{CONCLUSÃO}

Com o fim da revisão, percebe-se que o objetivo do estudo foi alcançado, uma vez que foram localizados artigos de intervenção em fisioterapia aquática bem como métodos avaliativos em solo e na água. Não foram identificadas, contudo, avaliações em ambiente aquático referentes a habilidades motoras de crianças. A única encontrada foi em relação à função e estrutura, não abrangendo todos os domínios da funcionalidade, incluindo atividades e participação. Por fim, este estudo explicita a necessidade de novas investigações sobre avaliação fisioterapêutica na intervenção em piscina aquecida.

\section{REFERÊNCIAS}

ARAUJO, L. B. et al. Análise eletromiográfica dos músculos tibial anterior e sóleo em pacientes hemiparéticos nos ambientes aquático e terrestre. Revista Brasileira Ciência e Movimento, v. 27, n. 3, p. 106-121, 2019.

BARBA, U. G. Avaliação da grade curricular e conhecimentos de residentes em pediatria sobre vigilância do desenvolvimento. 2007. Tese (Doutorado em Educação Especial) Universidade Federal de São Carlos, São Carlos, SP, 2007. 
BARBOSA, A. D. et al. Avaliação fisioterapêutica aquática. Fisioterapia em Movimento, v. 19, n. 2, p. 135-147, 2006.

BRAGA, H. V. et al. Efeito da fisioterapia aquática na força muscular respiratória de crianças e adolescentes com Síndrome de Down. Arquivos de Ciências da Saúde da Unipar, v. 23, n. 1, p. 9-13, 2019.

BVS. Biblioteca Virtual em Saúde [internet]. São Paulo: Departamento de Evidência e Inteligência para Ação em Saúde - ElH. 1998. Disponível em: https://bvsalud.org/. Acesso em: 18 maio 2020.

$\mathrm{CHOI}, \mathrm{H}$. Effects of therapeutic Tai chi on functional fitness and activities of daily living in patients with Parkinson disease. Journal of Exercise Rehabilitation, v. 12, n.5, p. 499-503, 2016.

GAVIM, A. E. O. et al. A influência da avaliação fisioterapêutica na reabilitação neurológica. Saúde em Foco, n. 6, p. 71-77, 2013.

GUERREIRO, T. B. F. et al. Triagem do desenvolvimento neuropsicomotor de crianças das Unidades de Educação Infantil do município de Belém, Pará, Brasil. Journal of Human Growth and Development. v. 26, n. 1, p. 181-189, 2016

HWANG, A. et al. Linkage of ICF-CY codes with environmental factors in studies of developmental outcomes of infants and toddlers with or at risk for motor delas. Disability and Rehabilitation, v. 36, n. 2, p. 89-104, 2014.

ISRAEL, V. L. Hidroterapia: proposta de um programa de ensino no trabalho com o lesado medular em piscina térmica. Fisioterapia em Movimento, v. 13, n. 1, p. 111-127, 2000.

ISRAEL, V. L.; PARDO, M. B. L. Hydrotherapy: Application of an Aquatic Functional Assessment Scale (Afas) in Aquatic Motor Skills Learning. American International Journal of Contemporary Research, v. 4, n. 2, p. 42-52, 2014.

IUCKSCH, D. D. et al. Decoding the aquatic motor behavior: description and reflection on the functional movement. Acta Scientiarum. Health Sciences, v. 16, n. 4, p. 335-340, 2020.

LAI, C. et al. Pediatric Aquatic Therapy on Motor Function and Enjoyment in Children Diagnosed With Cerebral Palsy of Various Motor Severities. Journal of Child Neurology. v. 30, n. 2, p. 200, 2015.

MÉLO, T. R. et al. Sistematização de instrumentos de avaliação para os dois primeiros anos de vida de bebês típicos ou em risco conforme o modelo da CIF. Fisioterapia e Pesquisa, v. 26, n. 4, p. 380-393, 2019.

MILLS, W. et al. Does Hydrotherapy Impact Behaviours Related to Mental Health and Well-Being for Children with Autism Spectrum Disorder? A Randomised Crossover-Controlled Pilot Trial. International Journal of Environmental Research and Public Health, v. 17, n. 2, p. 558, 2020.

NAZARIO, P. F.; ARINS G. C. B.; KURZ, G. H. Desenvolvimento motor e os movimentos reflexos. Revisão de literatura. EF Deportes - Revista Digital, n. 158, p. 16-158, 2016.

NOVAKOSKI, K. R. M. et al. De volta ao meio líquido: efeitos da intervenção de fisioterapia aquática realizada em prematuros. Rev. Bras. Cineantropom Hum., v. 20, n. 6, p. 566-575, 2018.

PLECASH, A. R.; LEAVITT, B. R. Aquatherapy for Neurodegenerative Disorders. Journal of Huntington's Disease, v. 3, n. 1, p. 5-11, 2014.

RAMALHO, V. M. et al. Protocolo de controle de tronco em ambiente aquático para crianças com paralisia cerebral: ensaio clínico randomizado. Rev. Bras. Ciênc. Saúde, v. 23, n. 1, p. 23-32, 2019.

SANTOS, C. P. A. et al. Fisioterapia aquática no tratamento de criança com distrofia muscular congênita merosina negativa: relato de caso. Acta Fisiátrica, v. 23, n. 2, p. 102-106, 2016.

SILVA, J. O. et al. Influência da estimulação aquática no desenvolvimento de crianças de 0 a 18 meses: um estudo piloto. Fisioterapia e Pesquisa, v. 16, n. 4, p. 335-340, 2009. 
SOUZA, M. T.; SILVA, M. D.; CARVALHO, R. Integrative review: what is it? How to do it?. Einstein, v. 8, n. 1, p. 102-106, 2010.

YAMAGUCHI, B. Programa de exercícios físicos aquáticos: efeitos nas habilidades motoras funcionais e qualidade de vida em pessoas com doença de Parkinson. 2016. Dissertação (Programa de Pós-Graduação em Educação Física) - Universidade Federal do Paraná, 2016. 